\title{
Los puertos del Pacífico mexicano en el contexto de la red global de transporte multimodal
}

\section{The Ports of the Mexican Pacific in the Context of the Global Multimodal Transport Network}

DOI: $10.32870 /$ mycp.v9i27.683

Carlos Martner-Peyrelongue ${ }^{1}$

\begin{abstract}
Resumen
A partir del proceso de apertura comercial iniciado en las últimas décadas del siglo $\mathrm{xx}$, los puertos modificaron paulatinamente sus funciones $y$ formas de articulación modal, logística y territorial para responder a los imperativos de alcance geográfico y eficiencia exigidos por cadenas productivas crecientemente globalizadas. En este sentido, en el presente trabajo se analiza la posición y el rol de los puertos de contenedores del Pacífico mexicano en el contexto de la conformación de una red global de transporte multimodal que condiciona y determina su destino. Desde una perspectiva conceptual basada en el espacio de flujos castelliano y el territorio de redes de Veltz, se evalúa la posibilidad de consolidar puertos concentradores en el Pacífico mexicano que articulen flujos no sólo desde y hacia México, sino también aquellos que tienen por origen o destino otros países de la región, potenciando, de esta manera, su relevancia como nodos portuarios clave de conexión de cadenas multimodales de transporte a nivel nacional y continental.
\end{abstract}

Palabras clave: globalización, Pacífico mexicano, puertos, geografía del transporte, redes multimodales.

\begin{abstract}
Since the starting of the process of commercial opening, initiated in the last decades of the 20th century, ports gradually modified their functions and forms of modal, logistic and territorial articulation to respond to the imperatives of geographical reach and efficiency demanded by increasingly globalized productive chains. In this sense, this work analyzes the position and the role of the container ports at the Mexican Pacific in the context of the formation of a global multimodal transport network that conditions and determines their destination. From a conceptual perspective based on the space of Castellian flows and the territory of Veltz' networks, it is evaluated the possibility of consolidating concentrating ports at the Mexican Pacific which articulate flows not only to and from Mexico, but also those that have their origin or destination at other countries in the region, thus promoting its relevance as key port nodes connecting multimodal transport chains at national and continental levels.
\end{abstract}

Keywords: globalization, Mexican Pacific, ports, transport geography, multimodal networks.

Artículo recibido el 21 de octubre de 2019 y dictaminado el 25 de mayo 2020.

1. Coordinador de Transporte Integrado y Logística en el Instituto Mexicano del Transporte y profesor en el Doctorado en Administración Marítima y Portuaria del Centro de Estudios Superiores Navales. Carretera El Colorado - Galindo Km. 12 Col. San Fandila, Pedro Escobedo, Querétaro, México. C.P. 76703 oRCID: https://orcid.org/0000-0002-7676-4256 Correo electrónico: martner@imt.mx 


\section{Introducción}

A partir del proceso de apertura comercial iniciado en la segunda mitad de la década de los ochenta del siglo pasado, los puertos han modificado paulatinamente sus funciones operativas, comerciales, logísticas y de vinculación territorial, dado que tienen que responder a una lógica distinta a la del periodo proteccionista previo, basado en el modelo de sustitución de importaciones. En efecto, actualmente tienen que responder a los imperativos de la globalización económica derivada del predominio de un modelo aperturista, donde se privilegió la desregulación y privatización de las actividades productivas, así como la liberación a escala global de los mercados tanto financieros, como de bienes y servicios, entre otros aspectos.

La fragmentación de la producción a escala planetaria para buscar ventajas comparativas en cada uno de los eslabones de los procesos de fabricación, la acelerada innovación tecnológica en los sistemas de comunicaciones y transportes, aunado al paulatino cambio del centro de gravedad del comercio internacional hacia la Cuenca del Pacífico, ha modificado sustancialmente el rol de los puertos, los cuales se están transformando en nodos clave de articulación logística, territorial y multimodal de extensas cadenas de suministro que trabajan con sistemas de proveeduría justo a tiempo o justo en secuencia (Cedillo, Lizárraga \& Martner, 2019).

En este sentido, la presente contribución tiene por objetivo analizar el desarrollo de los puertos mexicanos que mueven carga contenerizada en el litoral del Pacífico dentro del contexto de la reestructuración de rutas marítimas y redes logísticas intermodales motivadas por la globalización de los flujos de mercancías y del creciente intercambio comercial entre los países de la Cuenca del Pacífico. En particular, se buscará identificar y analizar la posición y el rol de los principales puertos del Pacífico mexicano dentro de la conformación de una red global de puertos y transporte multimodal, así como evaluar de manera global la posibilidad de consolidar puertos concentradores (conocidos como hubs a nivel internacional) que articulen tanto los flujos desde y hacia México, como aquellos que tienen destino final en otros países de la región, léase Centroamérica y Sudamérica principalmente.

Para avanzar en el planteamiento de tipo explicativo propuesto, se parte de una revisión crítica del modelo de puertos concentradores, adoptado de los países desarrollados sin análisis previo en las naciones emergentes. Metodológicamente se apoya en la conceptualización de un nuevo paradigma socio- 
territorial, cuyo elemento distintivo se encuentra en la conceptualización del espacio de redes y flujos (Veltz, 2006, 2014; Castells, 2010), para interpretar las nuevas configuraciones territoriales y dinámicas espaciales derivadas de los procesos de apertura comercial y la globalización.

A diferencia de los autores que han desarrollado el concepto de puertos y redes marítimas internacionales bajo las categorías de la ortodoxia dominante, donde el análisis del espacio global se realiza desde lo local o regional, el aporte conceptual propuesto consiste en ubicar la unidad de análisis en la conformación de un espacio global, al estilo del sistema-mundo capitalista marcado por tendencias polarizantes, que históricamente han sobrepasado los esfuerzos de convergencia entre las naciones (Wallerstein, 1996, 2016), donde las relaciones jerárquicas y asimétricas se manifiestan crecientemente a través de redes y flujos materiales e inmateriales (Castells, 2006; Veltz, 2014). Tal traslado de la unidad de análisis, poco valorado y comprendido por la corriente económica dominante (de corte neoclásico), permite revelar estructuras, configuraciones, dinámicas y tendencias que pasan inadvertidas para los países y las regiones y, por lo tanto, no son consideradas a la hora de establecer políticas públicas de desarrollo de infraestructura y tecnología.

Un ejemplo evidente de tal desconsideración es la multiplicación de proyectos para construir grandes obras de infraestructura de transporte (megapuertos, megaterminales, corredores internacionales bioceánicos, etc.) en cada uno de los países de Latinoamérica, cuestión que surge de manera casi natural cuando se ubica la unidad de análisis en el Estado-nación o en escalas menores, dentro de un entorno de incremento de los flujos, debido a la apertura comercial y la globalización en curso. Sin embargo, más allá del dato evidente, esto impide visualizar una estructura más profunda, como es la configuración de una red y un espacio global de flujos, cuya lógica de funcionamiento no es la misma que la de los Estados nacionales, puesto que, como señala Hiernaux (1995), la globalización de los procesos productivos y sociales no requiere el control del espacio continuo para operar, logra su expresión en puntos selectos - place o lugares a la Giddens - adecuadamente vinculados o articulados en red.

Por ello, la visión de tipo generalista o universalista que postula ortodoxia económica de inspiración neoclásica (neoliberal), cuyo modelo es eventualmente aplicable en cualquier lugar bajo la estandarización de ciertas condiciones técnicas, operativas y regulatorias, presenta serias limitaciones cuando pretende ser implantada en espacios periféricos de la economía-mundo 
capitalista y requiere de la incorporación de otros puntos relevantes en el análisis, como la ubicación del lugar, nodo o hub dentro del sistema global, su posición en el contexto de los principales ejes y corredores multimodales internacionales, la vinculación con los bloques económicos hegemónicos y las formas de articulación territorial de esos nodos con las regiones interiores, entre otros aspectos geoeconómicos a considerar.

Por el contrario, la presente propuesta plantea que el desarrollo de puertos concentradores o hubs no puede entenderse sin la caracterización de lo que hemos denominado como una red global de puertos y transporte multimodal, estructurada y definida por su alineación en torno a los tres bloques económicos dominantes en la actualidad, con ejes y corredores internacionales que determinan y permiten reconocer con mayor claridad el impacto en el desarrollo portuario de los litorales mexicanos y, eventualmente, en otros países latinoamericanos, cuya posición en la economía global dista mucho de ser hegemónica.

Este enfoque metodológico se complementa con la propuesta de criterios de análisis que ayuden a determinar las potencialidades y/o límites de los puertos del Pacífico mexicano en un contexto conducido por la formación de redes y corredores multimodales globales señalados previamente. El esquema de análisis propuesto no sólo retoma indicadores típicos en la evolución de un puerto, como la tasa de crecimiento de los flujos de carga y de los servicios de transporte marítimo, sino que incorpora nuevos elementos de análisis, relacionados con la geografía del transporte y la articulación logística, modal y territorial de los puertos.

Por tal razón, aquí se resalta el papel de la conexión espacial del puerto como fuente de obtención de carga y, por ende, la problemática de la integración modal cobra mayor relevancia. También se incorporan análisis de la posición de los puertos en función de los principales ejes del comercio marítimo internacional y de la combinación de servicios que incluyen el transbordo, tanto a puertos nacionales del Pacífico como de otros países del continente. Tales criterios proporcionan nuevas perspectivas de análisis sumamente necesarias para determinar el potencial de los puertos mexicanos a nivel continental y global.

Por último, se presentan perspectivas y conclusiones generales sobre el potencial para desarrollar puertos concentradores en México, en el contexto de la reestructuración de rutas y redes intermodales motivadas, entre otras cosas, por la creciente importancia económica y comercial del Pacífico mexicano. 
Los puertos del Pacífico mexicano en el contexto de la red global de transporte multimodal

\section{Globalización y cambio tecnológico en la formación de una red global de transporte multimodal}

\subsection{Hacia un modelo jerarquizado de puertos concentradores y alimentadores}

Para comprender la lógica de conformación de una red global de puertos y transporte multimodal se tienen que considerar al menos dos elementos fundamentales del proceso de globalización en curso, el cual no está exento de contradicciones y cuestionamientos. Por una parte, se encuentra la creación de las condiciones técnico-organizativas para integrar los procesos productivos crecientemente fragmentados en el espacio (Santos, 2000) debido a la deslocalización de las diversas fases de fabricación en ámbitos geográficos distantes y diferenciados y, por otra, la existencia de grandes bloques dominantes de la economía mundial que determinan en buena medida la densidad y direccionalidad de los flujos comerciales.

Efectivamente, la globalización de los procesos productivos ha supuesto su fragmentación espacial con la finalidad de encontrar ventajas comparativas para cada una de las fases de fabricación de un producto. Paralelamente, las innovaciones tecnológicas recientes, sobre todo, en los medios de comunicación y transporte, han apoyado esta tendencia de desconcentración productiva. El comercio internacional se ha transformado crecientemente en desplazamiento de bienes entre plantas de la misma empresa (flujos intrafirma) o entre el gran consorcio multinacional y sus subcontratistas internacionales que elaboran partes o insumos del producto final, para lo cual ha sido preciso implementar extensas redes que se desdoblan sobre espacios selectos del planeta, estructurando las nuevas relaciones de la economía mundial en lo que Castells (2006) ha llamado un espacio de flujos que se sobrepone a los vínculos de continuidad y cercanía de los espacios tradicionales, al integrar nodos territoriales, ampliamente separados en tiempo y espacio, en una red de producción-distribución extensa que puede operar de manera simultánea e integrada a grandes distancias.

Lo cierto es que las necesidades de este proceso de internacionalización derivaron en un nuevo modelo técnico-organizativo del transporte, basado en sistemas integrados multimodales y en el desarrollo de servicios logísticos puerta a puerta; en este entorno emergió el llamado modelo de Hub and Spoke (Rodrigue, 2017) como elemento central para estructurar una red global de puertos y transporte multimodal que permitiera efectivamente acelerar la 
circulación y crear fluidez mediante la articulación espacial de la fragmentación de las cadenas productivas de la globalización. La construcción del concepto de puerto hub (puerto concentrador, en español) proviene de los investigadores de países desarrollados dentro de la economía-mundo (Fosey, 1997; O'Kelly, 1998; Notteboom, 2008; Rodrigue, 2017) y, sin duda, refleja los cambios recientes que han sufrido en tecnología, en infraestructura y en organización los principales puertos a nivel internacional durante las últimas décadas.

El puerto concentrador o hub es concebido como una plataforma logística que concentra grandes volúmenes de carga e información, procedente de diversos espacios continentales, los cuales son procesados, organizados y distribuidos hacia distintas regiones, por tierra o por mar, mediante una eficiente articulación modal y territorial de los sistemas de transporte que convergen en el nodo portuario (Hoffmman, 2000; Notteboom, 2008).

Las dos vías para que un puerto concentre mayores volúmenes de carga son: la expansión de su hinterland y el desarrollo de actividades de transbordo de un buque a otro. La primera tiene que ver con el lado terrestre, es decir, con la expansión de la llamada zona de influencia territorial o hinterland mediante el desarrollo de potentes corredores intermodales que conecten al nodo portuario con regiones interiores cada vez más amplias y diversificadas. La segunda se refiere a la conectividad marítima (el foreland) que permite a un puerto no sólo concentrar y distribuir flujos de carga destinado a las regiones interiores del país de pertenencia, sino que puede servir también de plataforma logística para traspasar contenedores entre un buque mayor, que atiende normalmente a las rutas principales, a otra embarcación menor que llevará la caja metálica a su destino final, generalmente en zonas de menor densidad de carga. A esto se le denomina transbordo y existen puertos que manejan un alto porcentaje de este tipo de flujos, denominados hubs de transbordo.

Por otra parte, no puede omitirse la existencia de grandes polos o bloques dominantes de la economía mundial. Desde los años noventa del siglo Xx, Ohmae (1991) definió la llamada triada del poder, constituida básicamente por tres bloques geoeconómicos: la Unión Europea, Norteamérica y el noreste de Asia (incluyendo recientemente a China). De esta manera, existe una dualidad en el proceso globalizador expresada, por un lado, en una creciente capacidad de expansión y dispersión de las actividades económicas apoyada fuertemente en las nuevas tecnologías y, por otra, la centralización de las actividades de comando, alta dirección, innovación y concentración del valor 
agregado en las llamadas ciudades globales (Sassen, 2003) ubicadas sobre todo en la triada del poder.

En términos geográficos, esto se expresa precisamente en una concentración del Producto Interno Bruto (PIB) en los tres polos económicos dominantes. Los datos del Banco Mundial (2019) son claros al respecto. En 2018, la riqueza generada por las regiones del Lejano Oriente (noreste asiático), Europa (incluyendo Rusia) y Norteamérica (Canadá y Estados Unidos) concentraron el 75\% del PIB mundial.

Es importante señalar que todos estos lugares se localizan entre el paralelo 30 y el 60 del hemisferio norte, lo cual tiene connotaciones muy relevantes en la conectividad interespacial del sistema-mundo capitalista actual, que se articula a través de extensos corredores multimodales internacionales cuyos nodos centrales son los puertos concentradores.

En efecto, al analizar el movimiento global de contenedores, se observa un comportamiento geográfico parecido al de la distribución de la riqueza. Los flujos entre naciones del hemisferio norte ubicadas arriba del paralelo 30 , en 2018 , concentraron el $70 \%$ de los contenedores, mientras que el $30 \%$ restante correspondió al resto del mundo, incluido México.

Asimismo, cabe señalar el desplazamiento del centro de gravedad de los flujos internacionales de mercancías desde el Atlántico hacia la Cuenca del Pacífico (figura 1). Este proceso, iniciado en la década de los ochenta y sostenido hasta la actualidad, se explica por el rápido crecimiento e internacionalización económica de los países del bloque asiático, donde destacaron Japón y Corea en primera instancia y China más recientemente, los cuales se han constituido en los principales productores mundiales de manufacturas (bienes intermedios y de consumo final) ligados a las cadenas de suministro crecientemente globalizadas. 


\section{Figura 1}

Movimiento de contenedores por bloques económicos, en millones de TEUs, 2018

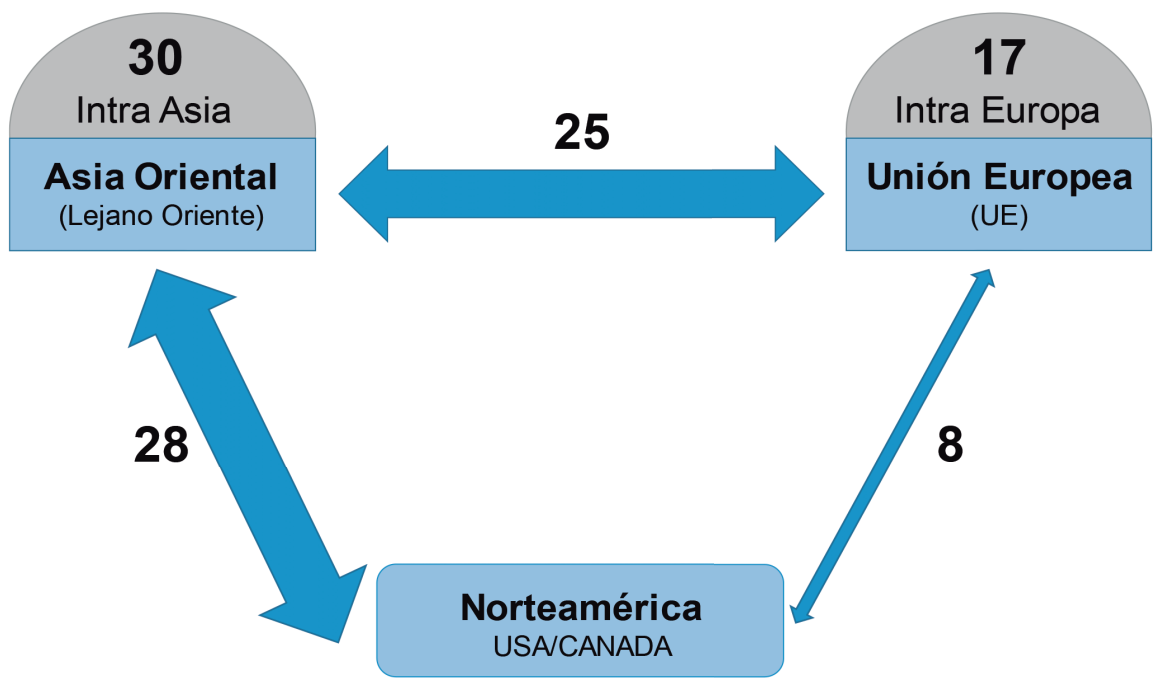

Nota: ${ }^{*}$ TEUs $=$ unidad de contenedor equivalente a 20 pies de largo.

Fuente: elaboración propia con base en datos de UNCTAD y Eurostat.

En efecto, la deslocalización de algunas fases del proceso productivo global hacia espacios selectos de la periferia o de lo que Sassen (2003) ha llamado el sur global, como los países del sudeste asiático, sur de China, la India, algunas regiones de México y de Latinoamérica, produce que la concentración de flujos de contenedores no sea tan acentuada como la concentración del PIB.

La alta densidad de los flujos entre los tres bloques dominantes constituye un eje este-oeste en el hemisferio norte que se articula con ejes de menor densidad en las rutas norte-sur, correspondientes al resto de los países del mundo, donde también operan segmentos de las cadenas de suministros globalizadas. En este contexto, los nodos clave que unen corredores y rutas de esta red global y ordenan los flujos de la producción fragmentada según sus orígenes y destinos, son precisamente los puertos concentradores.

En otras palabras, la dispersión de las cadenas de suministro, que mueven sobre todo bienes intermedios de un proceso productivo espacialmente segmentado, requiere de centros de control (hubs o concentradores) que enlacen 
de manera eficaz la superficie líquida y sólida del planeta y que concentren, ordenen y distribuyan los flujos permitiendo una integración coherente de la dispersión a través del desarrollo de extensas rutas y corredores de transporte regionales e intercontinentales de transporte multimodal.

Por ello, más allá de su definición y de la idea generalizada de que los hubs o concentradores pueden desarrollarse en las zonas costeras de cada nación (simplemente siguiendo los preceptos establecidos por políticas de apertura a iniciativas del capital privado y/o a esquemas público-privados), se requiere también un análisis geográfico que parte de sus atributos y localización frente a una compleja red continental e internacional de transporte multimodal.

\subsection{Concentradores globales y puertos alimentadores}

La aparición de grandes puertos concentradores (hubs) fue posible en la medida en que el tamaño de los buques creció y los operadores marítimos formaron grandes consorcios y/o alianzas. Sin embargo, para concentrar carga en un nodo portuario principal se requiere redefinir las funciones de varios puertos intermedios y al mismo tiempo es preciso desarrollar una estructura interconectada y jerárquica de puertos menores que suministren carga a los hubs (Martner, 2010).

Bajo este enfoque, se vuelve conceptualmente clara la idea de una red global de puertos y transporte multimodal. El esquema en cuestión implica que muchos puertos quedan excluidos de los servicios regulares directos $y$, en el mejor de los casos, podrán integrarse a la red mediante rutas alimentadoras (conocidas también como rutas feeders), las cuales conducen la carga, en embarcaciones menores, hacia algún concentrador donde será transbordada a los grandes buques para transportarla hasta su destino final.

De acuerdo con este planteamiento conceptual, en la cima de esta red se encuentran los concentradores globales, en virtud de la envergadura de las terminales y de los flujos, del tipo de embarcaciones operadas y de la cobertura geográfica que supone vínculos multicontinentales. Estos concentradores o hubs globales se localizan generalmente en el hemisferio norte, en las rutas este-oeste donde se concentran los principales corredores y redes de transporte marítimo.

No obstante, para que la red alcance mayor eficacia en la vinculación de flujos de diversa densidad, se necesita de nodos intermedios que faciliten el acceso a espacios continentales o subcontinentales como puntos de arti- 
culación entre corredores principales y rutas de menor densidad. Llamados indistintamente concentradores regionales o hubs intermedios (Rodrigue, 2017; Martner, 2010), estos nodos han adquirido una creciente importancia en las últimas décadas a medida que la fragmentación de las cadenas de suministro se expandió, alcanzando a un mayor número de países no centrales, denominados como economías emergentes.

\subsection{Concentradores regionales o intermedios}

"En efecto,..., en espacios emergentes, e históricamente no centrales dentro de la economía-mundo capitalista, caracterizados por fuertes incrementos recientes en los flujos comerciales, la red global ha requerido una instancia intermedia entre el concentrador global y los puertos alimentadores" (Martner, 2010 p. 330). Por eso, hay una sólida tendencia hacia la constitución de concentradores regionales, llamados también hubs intermedios por diversos autores (Rodrigue \& Notteboom, 2010), cuya dimensión, capacidad de carga y cobertura geográfica no es tan grande como la de los globales, pero abarcan ámbitos espaciales de cobertura notablemente más amplios que los de los puertos alimentadores.

Los concentradores regionales tienen un importante papel como nodos articuladores de espacios y redes logísticas, cuando logran explotar eficientemente la zona de influencia territorial o hinterland del puerto mediante la conexión intermodal con lugares de tierra adentro de un país o área continental específica, así como cuando son capaces de articular una fuerte conexión con las zonas de ultramar (foreland), a través de los servicios de transbordo entre un barco mayor y otro menor con la finalidad de acceder al destino final, cuando éste se localiza en rutas, corredores y/o espacios geográficos de menor densidad de flujos comerciales.

En la primera vertiente, algunos concentradores regionales o intermedios, generalmente ubicados en lugares selectos de países emergentes, tienden a modificar cualitativamente los vínculos territoriales, mediante la extensión de redes hacia una diversidad de zonas o territorios interiores que, en periodos previos, no tenían enlaces adecuados con los puertos en cuestión.

En la segunda vertiente, es decir, en la del foreland, se observa que los hubs regionales o intermedios se localizan principalmente en la intersección de los corredores este-oeste con las rutas norte-sur (Martner, 2008). Tal es el caso de las terminales portuarias de Balboa y Manzanillo (MIT), en Panamá, y 
de algunos puertos caribeños, como Kingston, en Jamaica, y Freeport, en Las Bahamas (figura 2). Estos concentradores vinculan básicamente, mediante el transbordo marítimo, flujos de gran diversidad de países del Caribe, Centro y Sudamérica con Asia, Europa y Norteamérica.

\section{Figura 2}

Principales concentradores globales y regionales, en millones de TEUs, 2013
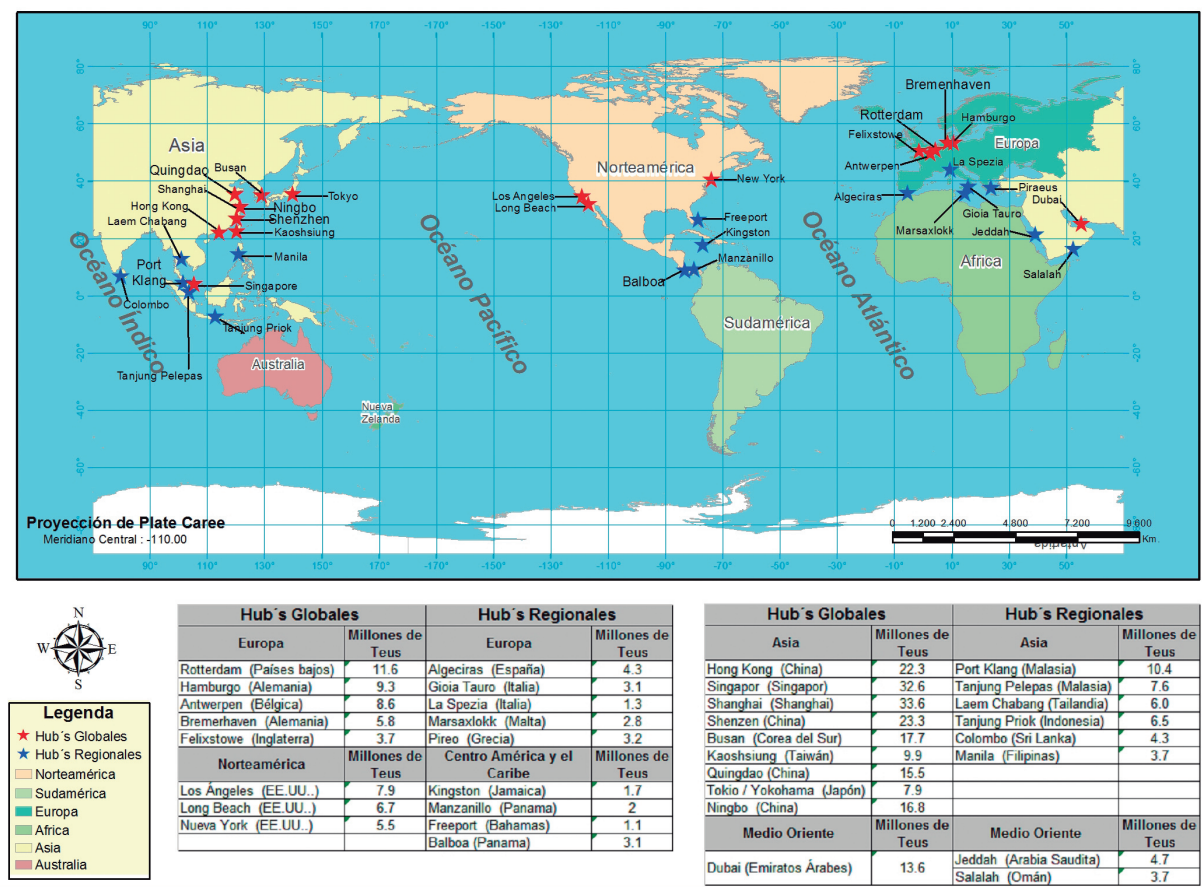

Nota: TEUs = unidad de contenedor equivalente a 20 pies de largo.

Fuente: elaboración propia con base en datos de Containerization International (2014).

Precisamente un elemento consustancial a la formación de la red global de puertos es la proliferación del transbordo. La segmentación de rutas y, con ello, el incremento de los servicios marítimos indirectos entre los diversos niveles jerárquicos de la red de puertos, es condición sine qua non para concentrar carga en estos puertos y para llenar buques cada vez más grandes. Entonces, el transbordo se constituye en un articulador de cadenas logísticas de producción y distribución. En otras palabras, con el transbordo se articula una 
red global de puertos y transporte multimodal, formada por concentradores de distinta envergadura y puertos alimentadores.

Desde luego, el modelo no es absoluto, en él conviven las rutas indirectas propias del modelo Hubs and Spoke con modalidades de servicio del modelo previo, llamado punto a punto (Point to Point) por diversos autores (Rodrigue, 2017), dado que sus itinerarios implican largas travesías intercontinentales en buques, generalmente de tamaño medio, que van recalando en un puerto tras otro en su trayecto, sin transbordos de contenedores en la ruta entre embarcaciones mayores y menores. Ahora bien, la elección o combinación de los modelos de servicio depende, en buena medida, de los volúmenes de carga en determinadas rutas, la densidad de los flujos, la capacidad de los puertos, los costos de transportación y distribución y las redes intermodales desarrolladas (Rodrigue \& Notteboom, 2011).

No obstante, en las últimas dos décadas el transbordo ha crecido vertiginosamente en el mundo, sin excepción de Latinoamérica y el Caribe. De hecho, los principales puertos de la región deben su dinamismo reciente a esta actividad. Tal es el caso de los puertos panameños, encabezados por los dos mayores concentradores latinoamericanos: Balboa, en el Pacífico, y el complejo portuario de Colón (Manzanillo MIT, CCT) y Cristóbal en el Atlántico. De 2003 a 2018, los puertos panameños pasaron de 1.6 millones de contenedores de transbordo de 20 pies (TEUs) a 6.1 millones de teUs (Autoridad Marítima de Panamá [AMP], 2019). Esto significa una acelerada tasa de crecimiento medio anual de $9.3 \%$, a pesar de los efectos negativos de la última crisis económica mundial. Cabe señalar que el $87 \%$ de los contenedores movidos por los puertos de Panamá son flujos transbordos que tienen como origen-destino países distintos a Panamá, sobre todo diversas naciones de Centroamérica, Sudamérica y el Caribe.

Lo propio puede decirse del puerto colombiano de Cartagena, de Kingston, en Jamaica, y de Freeport, en Bahamas, entre otros. Por su parte, en México destaca recientemente el acelerado crecimiento del transbordo en los principales puertos del Pacífico: Manzanillo y Lázaro Cárdenas, cuestión que se analizará con más detalle en el siguiente apartado.

De hecho, es materia primordial de este trabajo establecer la viabilidad de los puertos mexicanos del Pacífico en el ámbito de las nuevas tendencias del transporte marítimo internacional, así como detectar sus potencialidades para constituirse en puertos concentradores intermedios por vía del transbordo y/o de la articulación intermodal con regiones interiores del país, en un con- 
texto donde la consolidación de la red de puertos y el eventual desarrollo y/o modernización de corredores intermodales internacionales atraerá mayores flujos y embarcaciones más grandes a la región latinoamericana.

Desde esta perspectiva, en los siguientes apartados se analizará el potencial de los puertos del Pacífico mexicano con base en criterios geoeconómicos relativos al despliegue de los sistemas de transporte sobre el espacio continental y el territorio nacional, entre los que destacan la ubicación de los nodos portuarios de este litoral frente a los principales ejes multimodales internacionales, el ámbito territorial de obtención de carga de los puertos del Pacífico mexicano y el desarrollo de corredores intermodales que vinculan a las principales zonas urbanas de producción y consumo del país con los puertos nacionales.

\section{Los puertos mexicanos del Pacífico frente a las redes y corredores intermodales globales}

\subsection{Los puertos del Pacífico mexicano frente a los principales ejes intermodales internacionales}

En una escala geográfica global, el potencial de los puertos para ascender dentro de la red global tiene que ser analizada en función de la ubicación de los principales ejes de transporte marítimo e intermodal, cuestión que determina, en buena medida, las posibilidades de constituir hubs regionales o globales dentro de la red. La distancia respecto a los principales corredores de transporte marítimo y multimodal internacional es un dato clave para el desarrollo de puertos concentradores, tal como lo demostró Hoffmann (2000) para el caso del litoral del Pacífico en Sudamérica.

Normalmente los grandes puertos concentradores se localizan en los corredores este-oeste del hemisferio norte, debido a la densidad de los flujos de mercancías que circulan entre los tres grandes polos económicos de la globalización, mientras que los concentradores intermedios o regionales encuentran mayor oportunidad de desarrollo en las zonas de confluencia entre las rutas verticales (sur-norte) y las horizontales (este-oeste).

En el hemisferio norte del continente americano, esto sucede principalmente en torno al Canal de Panamá, donde se localizan importantes hubs intermedios de transbordo, tales como Balboa PPC y PSA Panamá en el Pacífico, así como Manzanillo MIT, Colón cт y Cristóbal PPC en el Atlántico. Sin duda 
el cruce interoceánico panameño extiende las posibilidades de intersección entre las grandes embarcaciones que provienen de los corredores este-oeste, con aquellas que se mueven en las rutas norte-sur de América.

La relevancia que tiene el enorme mercado de la costa este de Estados Unidos para los productos asiáticos procedentes del Lejano Oriente (principalmente de China, Corea y Japón) impone la condición de que las embarcaciones tengan que bajar hasta el paralelo 9 para cruzar del Pacífico al Atlántico vía el Canal de Panamá. Esta peculiaridad abre la posibilidad de expandir un vasto eje de intersección entre los flujos este-oeste y los norte-sur, que desciende desde las costas oeste y este de Estados Unidos hasta el istmo panameño (figura 3). Precisamente esto explica el desarrollo de los concentradores regionales de transbordo en Panamá y en otros puertos de la región ubicados cerca de estos ejes de intersección, como sucede en el Caribe con casos como los de Kingston, Freeport y Cartagena (figura 3).

En este punto cabe preguntarse dónde se ubican los puertos mexicanos respecto a la condición geográfica generada por los ejes de intersección de rutas. Lo primero que se aprecia es que las condiciones son muy distintas dependiendo del litoral del que se trate. En efecto, los puertos del Golfo tienen desventajas por encontrarse relativamente distantes del eje de intersección del Atlántico, lo cual implica entre tres y cuatro días adicionales de navegación para los buques de largo itinerario con rutas desde el Lejano Oriente hacia la costa este estadounidense, por lo que difícilmente serán escogidos como concentradores regionales de transbordo (Martner, 2010).

Por el contrario, los principales puertos del Pacífico mexicano, Manzanillo y Lázaro Cárdenas, se encuentran mejor posicionados para acceder a la categoría de concentradores regionales de transbordo de contenedores, puesto que ambos se localizan muy cerca de la vertiente pacífica del citado eje de intersección de rutas (figura 3) y ambos han desarrollado un amplio hinterland nacional, al concentrar cada vez más flujos de carga contenerizada generados o atraídos por múltiples regiones de producción y consumo del país, tal como se señaló previamente. 


\section{Figura 3}

Puertos mexicanos en el contexto

de los principales ejes de transporte multimodal

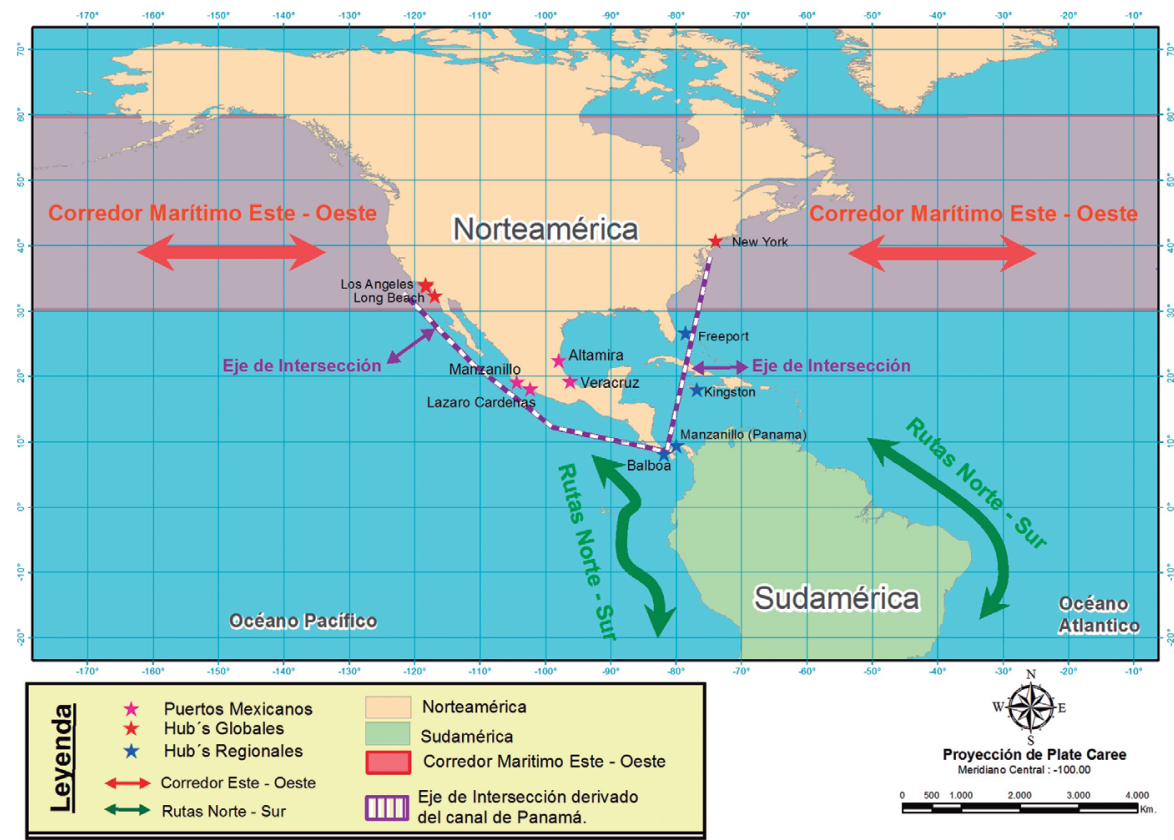

Fuente: elaboración propia.

3.2. El ascenso de Manzanillo y Lázaro Cárdenas como concentradores regionales en el Pacífico

En las últimas décadas Manzanillo y Lázaro Cárdenas se han convertido en los puertos más importantes de México en el movimiento de contenedores. Entre el año 2000 y 2019 el puerto de Manzanillo tuvo una tasa de crecimiento promedio de $11 \%$ anual en el flujo de contenedores para alcanzar durante el último año una cifra superior a los tres millones de TEUs. Por su parte, entre 2007 y 2019 Lázaro Cárdenas tuvo un crecimiento promedio de 14\% anual para alcanzar 1.3 millones de TEUs durante los últimos 12 meses (Coordinación General de Puertos y Marina Mercante [CGPMM], 2020).

Sin duda ambos han mantenido un elevado dinamismo en las dos primeras décadas del siglo XXI y parecen estar en condiciones de escalar dentro de 
la red global de puertos hasta constituirse en hubs regionales o intermedios, dado que obtienen carga no sólo de su hinterland cada vez más amplio dentro del territorio nacional, sino también de la interconexión marítima y las operaciones de transbordo.

Así ha sucedido desde el año 2005, cuando ambos puertos comenzaron a incrementar sensiblemente este tipo de operaciones. Entre 2005 y 2018 Manzanillo quintuplicó el número de contenedores de transbordo al pasar de 200 mil teUs a más de un millón 100 mil TEUs, lo cual supuso una tasa de crecimiento promedio de $13.8 \%$ anual (figura 4). En el mismo periodo, Lázaro Cárdenas pasó de 24 mil a 396 mil teUs de transbordo, con una impresionante tasa media de crecimiento de $24 \%$ anual en este rubro.

Los datos previamente analizados revelan que una parte sustancial del dinámico crecimiento de ambos puertos se debe no solamente al incremento del comercio exterior mexicano, sino a la emergencia de actividades propias de los hubs regionales, los cuales van más allá de su propio mercado (en este caso, el mexicano) para alcanzar mercados en un ámbito continental o, al menos subcontinental, es decir, para fungir como plataforma logística de concentración y distribución de flujos desplegadas en vastos espacios y territorios del continente, más allá de las cadenas productivas y logísticas vinculadas al comercio exterior mexicano, hasta alcanzar relevancia como nodos articuladores de flujos de bienes con origen y/o destino en Centro y Sudamérica.

En efecto, Manzanillo y Lázaro Cárdenas se constituyen en articuladores de redes de transportación de bienes de cadenas productivas, principalmente entre Asia y países latinoamericanos con puertos en la fachada marítima del Pacífico. Dentro del modelo de puertos concentradores y alimentadores (hubs and spoke, en idioma inglés) esto significa que los contenedores viajan en embarcaciones de gran tamaño desde el Lejano Oriente hasta los mencionados puertos mexicanos, donde un $64 \%$ de éstos serán descargados y distribuidos en el territorio nacional, mientras que el $36 \%$ restante serán transbordados a embarcaciones menores para llevarlos a diversos países del continente. 


\section{Figura 4}

Evolución de los flujos de contenedores de transbordo en el puerto de Manzanillo, México, 2005-2018

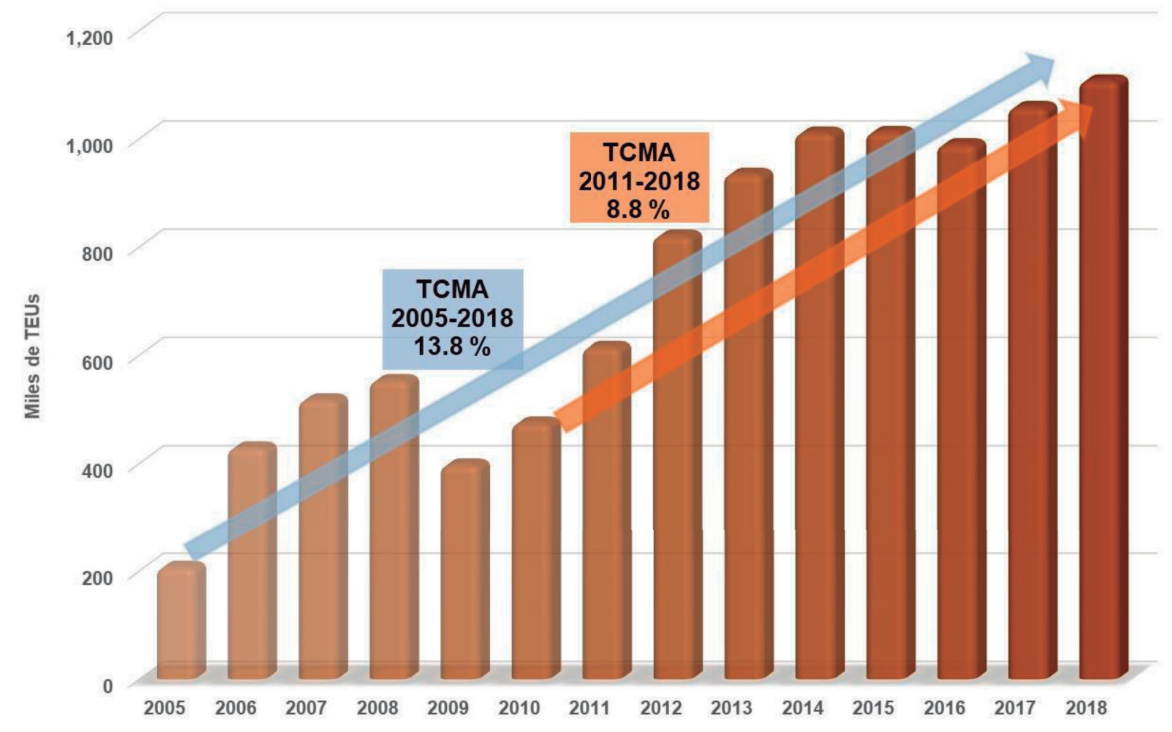

TCMA: tasa de crecimiento medio anual.

Fuente: elaboración propia con datos de la CGPMM-SCT (2019).

Tal como se observa en la tabla 1, Lázaro Cárdenas opera también como nodo articulador de cadenas que realizan el transbordo de carga movida en contenedores entre Asia y diversos países latinoamericanos, predominando aquellos que tienen puertos en litoral del Pacífico. Notoriamente destaca la región centroamericana, con flujos muy significativos vinculados a Guatemala, a través de Puerto Quetzal; a El Salvador mediante el puerto de Acajutla; a Costa Rica vía Puerto Cadera, a Nicaragua por el Puerto de Corinto, a Honduras por San Lorenzo y a Panamá a través de Balboa. Asimismo, en el puerto michoacano se articulan flujos de transbordo con origen o destino en países sudamericanos de la Cuenca del Pacífico como Chile, Perú, Colombia y Ecuador. 
Tabla 1

Carga contenerizada con origen-destino en América Latina que realiza transbordo de buques en el Puerto de Lázaro Cárdenas, 2018

(en toneladas)

\begin{tabular}{|l|r||l|r|}
\hline $\begin{array}{c}\text { Desde Asia a Centro y } \\
\text { Sudamérica }\end{array}$ & \multicolumn{1}{|c|}{ Toneladas } & \multicolumn{1}{|c|}{$\begin{array}{c}\text { Desde Centro y } \\
\text { Sudamérica a Asia }\end{array}$} & Toneladas \\
\hline Guatemala & 340,114 & Guatemala & 129,647 \\
\hline El Salvador & 215,331 & Nicaragua & 109,387 \\
\hline Chile & 171,369 & Costa Rica & 87,262 \\
\hline Perú & 131,504 & El Salvador & 75,479 \\
\hline Nicaragua & 116,633 & Panamá & 57,024 \\
\hline Honduras & 102,490 & Perú & 52,713 \\
\hline Costa Rica & 82,258 & Chile & 51,252 \\
\hline Panamá & 75,827 & Colombia & 15,908 \\
\hline Colombia & 68,608 & Honduras & 4,000 \\
\hline Ecuador & 53,170 & Ecuador & 943 \\
\hline $\begin{array}{l}\text { Otros países de A.Latina } \\
\text { y El Caribe }\end{array}$ & 20,183 & $\begin{array}{l}\text { Otros países de A.Latina } \\
\text { y El Caribe }\end{array}$ & 11,013 \\
\hline Total & $1,377,487$ & Total & 594,628 \\
\hline
\end{tabular}

Fuente: elaboración propia con base en datos del Anuario estadístico del movimiento portuario (2018), de CGPMM-SCT.

Ahora bien, es importante señalar que el creciente protagonismo de Manzanillo y Lázaro Cárdenas en el litoral del Pacífico resulta tanto del ascenso del transbordo como del incremento de los flujos con origen o destino en México, de tal forma que dichos puertos concentran carga por estas dos vías y ello los fortalece dentro de la red global de transporte multimodal.

Tal como en su momento concluyeron Zohil y Prijon (1999) al analizar la relación entre el tráfico de contenedores generado por el hinterland portuario y el de transbordo en los puertos del Mar Mediterráneo, al señalar que: 
[...] los volúmenes de tráfico de transbordo de contenedores en un puerto son una función lineal del volumen de tráfico portuario [generado por el hinterland], y una función lineal inversa de la distancia de la ruta principal de tránsito. En otras palabras, los barcos tienden a preferir a los puertos para los cuales ellos tienen carga local y aprovechan que ya están allí para realizar movimientos de transbordo. Cuanto menos desvío de la ruta principal implique la escala, más alta es la probabilidad de que se le elija como centro de transbordo. (Zohil y Prijon, 1999 p. 185)

En el Pacífico mexicano, Lázaro Cárdenas y Manzanillo están en sintonía con estos supuestos y han avanzado durante la última década hacia la categoría de puertos concentradores regionales en la medida en que están aumentado significativamente el movimiento de carga contenerizada al ampliar su zona de influencia territorial con regiones interiores cada vez más distantes, además de encontrarse geográficamente muy cerca del eje de intersección de rutas que baja desde la costa oeste de Estados Unidos hasta el Canal de Panamá. Por lo tanto, las ventajas comparativas están presentes y tendrán que ser aprovechadas mediante la acción de los actores económicos vinculados a los puertos en cuestión.

No obstante, para consolidar tal proceso en el largo plazo es necesario establecer objetivos de planeación para ganar posiciones ante la fuerte competencia de otros puertos de la región que buscan concentrar carga (Martner, 2010) y convertirse en concentradores regionales o intermedios, como los puertos de la zona de Balboa, en Panamá; Puerto Quetzal, en Guatemala y Puerto Caldera, en Costa Rica.

Ahora bien, para que los puertos mexicanos fortalezcan su posición dentro de la red global y aprovechen las oportunidades tecnológicas y operativas de los modernos sistemas de transporte, se requiere no sólo mejorar las cualidades y capacidades del nodo portuario, sino de toda la red multimodal y, en especial, de la conectividad terrestre con el hinterland y/o el ámbito territorial desde donde obtiene la carga, cuestión que se abordará en los siguientes incisos.

\section{3. Ámbito territorial de obtención de carga de los puertos del Pacífico mexicano}

Tradicionalmente los puertos obtenían la totalidad de la carga de su región contigua, es decir, los flujos de mercancías eran atraídos o generados mayoritariamente por las localidades y centros urbanos cercanos al puerto. La zona de influencia territorial o hinterland estaba muy acotada por la cercanía 
física, debido a la escasa eficiencia operativa de los puertos, a las barreras regulatorias y a las dificultades de acceso del transporte terrestre. En este sentido, los expertos en temas portuarios de la Conferencia de las Naciones Unidas sobre Comercio y Desarrollo (UNCTAD, 1992) decían que los puertos latinoamericanos tenían hinterlands cautivos, puesto que estas limitaciones de accesibilidad, regulación y eficiencia operativa constituían trabas muy costosas para que un puerto compitiera por el mercado ubicado dentro de la acotada zona de influencia de otro puerto.

Por el contrario, con el desarrollo del intermodalismo y la superación de trabas legales, administrativas y operativas, el ámbito espacial para atraer o generar carga se amplió notablemente. Asimismo, los hinterlands cautivos se rompieron a favor de un hinteland común que puede ser disputado y compartido por varios puertos simultáneamente, siempre y cuando la integración multimodal de los modos de transporte marítimos y terrestres lo permita.

Por ejemplo, los puertos estadounidenses del Pacífico y del Atlántico han expandido notablemente su hinterland gracias al desarrollo de los sistemas de transporte intermodal. Ahora incluso disputan la zona de influencia tradicional de los puertos mexicanos y canadienses. Por eso, en la actualidad una de las principales formas para concentrar carga y transformar a un puerto en concentrador intermedio o global es ampliando su zona de influencia territorial, es decir, expandiendo su hinterland para abarcar regiones tierra adentro que antes resultaban inalcanzables dadas las carencias e ineficiencias en la operación y la infraestructura de transporte.

Otra forma de concentrar carga desde ámbitos espaciales lejanos es el desarrollo del transbordo marítimo. Tal como se señaló en los apartados previos, un creciente número de puertos en el mundo se han convertido en concentradores intermedios de transbordo, incluso algunos viven casi exclusivamente de esta actividad, tan propia del reciente esquema de red global de puertos.

Ahora bien, para conocer el potencial de los puertos del Pacífico mexicano y las probabilidades de incorporarse y escalar posiciones dentro de la red global de puertos es preciso, en cada caso particular, analizar el ámbito espacial desde el cual obtienen su carga. Para ello se utilizó el criterio de identificación de la zona de influencia territorial o hinterland de cada puerto.

Los puertos que tienen un hinterland limitado a su región más próxima (hinterland tradicional) difícilmente ascenderán en la jerarquía de la red global; en cambio, los que logren ampliar su hinterland mediante el desarrollo 
de corredores intermodales y/o incorporen actividades de transbordo podrán concentrar carga y alcanzar posiciones más sólidas en la red.

En este sentido, el desarrollo de infraestructura hacia puntos selectos del litoral y la creación de cadenas logísticas integradas en las que intervienen actores con presencia global, propician la desaparición de los hinterland cautivos y, por lo tanto, modifican la importancia relativa de los puertos.

Los puertos más dinámicos, al ampliar su vinculación territorial, invaden y disputan la zona de influencia de puertos vecinos, otrora cautiva. Por lo tanto, el multimodalismo y las mejoras físicas en la accesibilidad territorial propician la formación de hinterlands comunes a muchos puertos, cada vez más competidos por los actores portuarios y, en general, por los operadores de transporte multimodal (Martner, 2010). Y es que, como señala Noteboom (2008), la batalla competitiva entre puertos cada vez más se librará en tierra. Por lo que las conexiones con el interior se han transformado en un área clave para la expansión y consolidación de los puertos.

En México se observa una notable transformación territorial a partir de la concentración de flujos y redes en determinados puertos. En efecto, la construcción de autopistas, redes de ferrocarril de doble estiba de contenedores, terminales multimodales, centros de distribución y zonas de actividades logísticas son obras de infraestructura que, por su propia envergadura, implican modificaciones territoriales de determinados fragmentos espaciales $y$ refuerzan las dinámicas territoriales puerto-interior.

Los desarrollos de tales infraestructuras propiciaron una enorme ampliación del hinterland de los principales puertos mexicanos (Manzanillo y Lázaro Cárdenas, en el Pacífico), permitiendo una vinculación multirregional (tabla 2) basada en el incremento de las conexiones intermodales y los servicios logísticos hacia las regiones interiores más dinámicas del país, como la megalópolis del Valle de México, la región del Bajío, el centro-occidente, el noreste y la frontera norte del país.

De esta forma, los dos puertos mencionados obtienen la carga de un hinterland ampliado, compuesto por diversas regiones en las cuales la cercanía física no es tan relevante. Se trata de una estructura de vinculación multirregional y diversificada que, eventualmente, permite al puerto concentrar elevados volúmenes de carga.

Por el contrario, el análisis de este criterio mostró que la mayor parte de los puertos mexicanos tienen escasas posibilidades de convertirse en puertos concentradores. En el litoral del Pacífico, los puertos Guaymas, Topolobampo, 
Mazatlán, Salina Cruz y Puerto Chiapas muestran un hinterland limitado, confinado a las zonas cercanas a los respectivos puertos. Aquí la cercanía física al puerto tiene un papel preponderante y las posibilidades de concentrar carga atraída de otras regiones del país son escasas, dada la ausencia de conexiones y servicios multimodales regulares.

\section{Tabla 2}

Ámbito espacial de obtención de carga contenerizada en los puertos del Pacífico mexicano

\begin{tabular}{|c|c|c|c|}
\hline Ámbito & Cobertura espacial & Conexión intermodal & Puertos \\
\hline $\begin{array}{l}\text { Hinterland } \\
\text { tradicional }\end{array}$ & $\begin{array}{l}\text { Limitada } \\
\text { Zonas contiguas al } \\
\text { puerto }\end{array}$ & $\begin{array}{l}\text { Escasa integración multimodal. } \\
\text { Baja densidad de flujos. } \\
\text { Escasa participación del } \\
\text { ferrocarril. } \\
\text { Carencia de servicios e } \\
\text { infraestructura especializada. } \\
\text { Oferta de servicios tradicionales. }\end{array}$ & $\begin{array}{l}\text { Ensenada } \\
\text { Guaymas } \\
\text { Mazatlán } \\
\text { Salina Cruz } \\
\text { Puerto Chiapas }\end{array}$ \\
\hline $\begin{array}{l}\text { Hinterland } \\
\text { ampliado }\end{array}$ & $\begin{array}{l}\text { Amplia } \\
\text { Multirregional }\end{array}$ & $\begin{array}{l}\text { Infraestructura y servicios } \\
\text { especializados. } \\
\text { Terminales intermodales. } \\
\text { Desarrollo de corredores } \\
\text { multimodales. } \\
\text { Trenes de doble estiba. } \\
\text { Operadores de transporte } \\
\text { multimodal. }\end{array}$ & $\begin{array}{l}\text { Manzanillo } \\
\text { Lázaro Cárdenas }\end{array}$ \\
\hline $\begin{array}{l}\text { Transbordo } \\
\text { marítimo }\end{array}$ & $\begin{array}{l}\text { Amplia } \\
\text { Nacional } \\
\text { Subcontinental }\end{array}$ & $\begin{array}{l}\text { Fuertes conexiones marítimas. } \\
\text { Nodo de enlace entre rutas } \\
\text { directas e indirectas. } \\
\text { Optimización de maniobras } \\
\text { portuarias. }\end{array}$ & $\begin{array}{l}\text { Manzanillo } \\
\text { Lázaro Cárdenas }\end{array}$ \\
\hline
\end{tabular}

Fuente: elaboración propia.

Efectivamente, en tales puertos los flujos tienden a ser poco densos y las redes o conexiones intermodales alcanzan un escaso desarrollo. Los agentes ofrecen los servicios tradicionales de carga, descarga y almacenamiento de mercancías, así como de avituallamiento de embarcaciones. En estas circunstancias hay escasez de servicios e infraestructura especializada, ausencia de servicios regulares de trenes contenedores de estiba sencilla o doble estiba y poca participación del modo ferroviario. No obstante, algunos de estos nodos 
tienen potencial para constituirse en eficientes puertos alimentadores dentro de la red global.

Dentro de los puertos alimentadores del Pacífico que presentan potencial para captar mayores volúmenes de carga y, eventualmente, convertirse en concentradores regionales de carga contenerizada, se encuentra el de Salina Cruz, Oaxaca. Como es del conocimiento público, este puerto forma parte del Istmo de Tehuantepec, región que desde hace décadas ha sido motivo de múltiples proyectos para desarrollar un corredor interoceánico entre el Océano Pacífico y el Golfo de México, atrayendo flujos de carga internacional movidos actualmente por el Canal de Panamá. Cabe señalar que ninguna de esas propuestas logró detonar el cruce interoceánico, tal como lo atestiguan múltiples estudios y evaluaciones sobre tales iniciativas (Segura \& Sorroza, 1994; San Martín, 1997; Instituto Mexicano del Transporte [IMT-SCT], 2002; Martner, 2012; Rodríguez, 2019).

En general, los estudios mencionados coinciden en la complejidad económica, técnica y operativa de los proyectos previos del corredor del Istmo, pero sobre todo remarcan la ausencia de una propuesta integral de desarrollo regional incluyente para consolidar actividades productivas locales, bajo una concepción de bienestar y sustentabilidad que privilegie iniciativas para impulsar modalidades de desarrollo endógeno sobre el desarrollo exógeno.

Del mismo modo, varios estudios identificaron que el eventual cruce interoceánico del Istmo no sólo competiría con el Canal de Panamá, también lo haría con los llamados puentes terrestres intermodales estadounidenses basados en eficientes trenes de doble estiba de contenedores que conectan los puertos con las principales ciudades y regiones interiores de ese país. Así, la posibilidad de que el puerto de Salina Cruz se convierta en un concentrador intermedio a nivel subcontinental pasa necesariamente por un proyecto de desarrollo regional de largo aliento, donde el crecimiento de la actividad económica en la zona constituya el principal elemento generador de flujos de carga tanto domésticos como de comercio exterior. Sin duda, ésta constituiría una garantía para consolidar puertos y corredores de transportes menos dependientes de los avatares de los flujos internacionales.

En este sentido, una manera consistente de reducir las desventajas que se tienen frente a otros cruces interoceánicos y corredores intermodales existentes (léase el Canal de Panamá ampliado y los puentes terrestres estadounidenses) pasa por generar carga en la propia región y densificar la ruta de manera endógena. Efectivamente, los buques son atraídos y consolidan 
rutas duraderas, sobre todo en aquellos lugares donde existe carga local y aprovechan tales escalas para transbordar contenedores o embarques que se dirigen a otros mercados o países, tal como se mencionó previamente. Entonces, la apuesta principal de largo plazo tendría que ser, antes que nada, por el desarrollo local y regional del Istmo de Tehuantepec, es decir, por la creación de actividades productivas que generen crecientes volúmenes de mercancías tanto para el mercado nacional, como para el internacional.

\subsection{Integración multimodal de los puertos mexicanos}

El ámbito espacial de obtención de carga contenerizada en los puertos mexicanos está íntimamente vinculado a la necesidad de desarrollar y consolidar corredores intermodales que los vinculen de manera eficaz, confiable y competitiva con las regiones interiores de producción y consumo del país.

Esto es fundamental para concentrar carga y reforzar la posición competitiva de los puertos mexicanos, sobre todo cuando la mayoría de la carga es generada o atraída por ciudades y regiones del interior del país. Sin embargo, la interfase marítimo-terrestre presenta todavía problemas de integración modal, facilitación y de coordinación de las diversas actividades vinculadas a la distribución física internacional de las mercancías.

En diversos trabajos (Martner, 2008; Zanela, 2015) se ha señalado el problema que representa el protagonismo desmedido que ocupa el circuito de las revisiones de la mercancía, en desmedro de la fluidez de las cadenas de comercio exterior. Una mejor coordinación y cooperación entre las instituciones que intervienen en esa fase, sin duda conduciría hacia el abatimiento de los tiempos muertos de inmovilización de las mercancías en las terminales portuarias, pero también las opciones de transporte terrestres tendrán que modernizarse, tanto en la operación como en la incorporación de estrategias logísticas de distribución y en la actualización tecnológica.

Por lo pronto, un dato crítico de la interfase marítimo-terrestre es el tiempo de estadía de los contenedores en puerto, cuyo promedio alcanzó los 6.8 días en el año 2018. Si bien es cierto que en esta fase concurren gran cantidad de actores y actividades relacionadas con la inspección, clasificación de la mercancía, pago de aranceles, revisión aduanera y disponibilidad de transporte terrestre, se requiere mejorar los procesos de facilitación y de coordinación de autoridades para el desalojo expedito de la carga, a fin de 
reducir sustancialmente este indicador y propiciar el desarrollo de cadenas de suministro eficientes a través de los puertos.

Otro dato importante se refiere a la conectividad de los corredores intermodales de trenes de doble estiba de contenedores. En este punto, nuevamente se observan avances importantes en los dos principales puertos del Pacífico, Manzanillo y Lázaro Cárdenas, mismos que han consolidado sólidos corredores intermodales de doble estiba de contendores hacia el centro y norte del país. En efecto, en estos casos se observan las conexiones multimodales de mayor densidad hacia regiones dinámicas del interior del país como el Bajío, la megalópolis del Valle de México, la región centro-norte y las zonas productoras del noreste y la frontera norte.

$\mathrm{Al}$ respecto, en la figura 5 se observan los resultados de un modelo de asignación de carga intermodal en México, donde se identifica, de manera jerarquizada, la importancia de los corredores intermodales que operan en el país en función de la densidad de flujos y la posición geográfica de los principales nodos y arcos de la red. 


\section{Figura 5}

Principales corredores intermodales en México, 2017

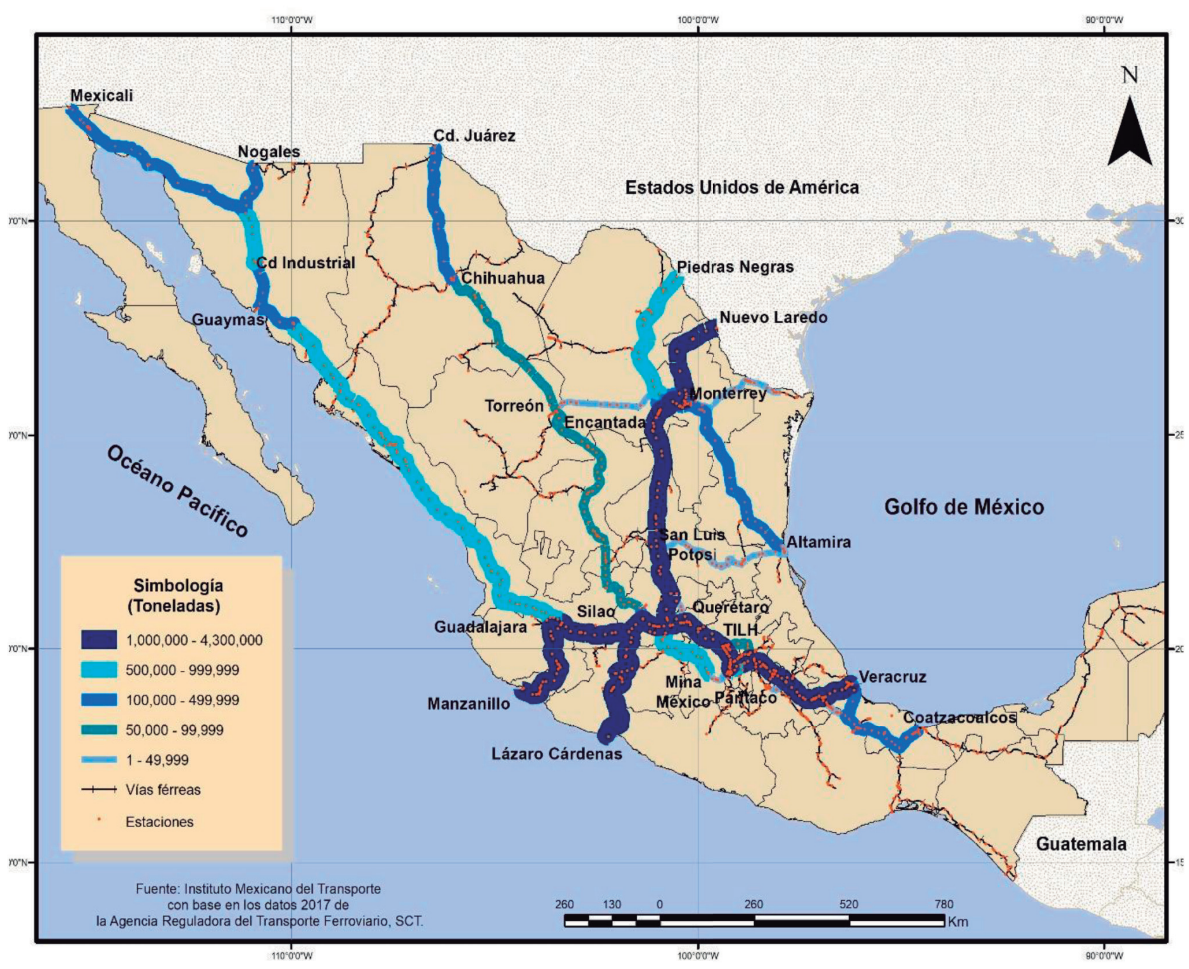

Fuente: elaboración IMT con datos de la Agencia Reguladora de Transporte Ferroviario (2018).

Este modelo de asignación aplicado a la red intermodal permite concluir que en México existen pocos corredores consolidados de alta densidad, que movilizan cifras superiores al millón de toneladas anuales. No obstante, cuatro de ellos son ejes transversales y van desde el litoral del Pacífico a los principales centros demográficos y económicos del país. Tales corredores abarcan los siguientes nodos y regiones: a) Manzanillo-Guadalajara-Bajío-Zona Metropolitana de la Ciudad de México; b) Lázaro Cárdenas-Querétaro-Zona Metropolitana de la Ciudad de México; c) Manzanillo-Guadalajara-Aguascalientes-Monterrey; d) Lázaro Cárdenas-San Luis Potosí- Monterrey.

Desde luego, también destaca la densidad del eje norte-sur que vincula la frontera norte con el centro del país en el corredor intermodal constitui- 
do por la ruta Nuevo Laredo-Monterrey-San Luis Potosí-Querétaro-Zona Metropolitana de la Ciudad de México, así como el corredor que vincula al puerto de Veracruz con el centro del país. En contraparte, la región del sursureste de México presenta débiles flujos intermodales y, por ende, ausencia de corredores y servicios intermodales establecidos de manera continua y eficiente, lo cual la ubica en una situación poco competitiva y desventajosa frente a otras zonas del país.

\section{Conclusiones}

El desarrollo de los puertos mexicanos del Pacífico no puede explicarse ni entenderse fuera del contexto de una red global de puertos y transporte multimodal, que es sustento físico, tecnológico y operativo de las necesidades de movilidad y externalización de la producción en la fase de globalización de la economía. Esta red, mediante sus nodos clave (los puertos concentradores o hubs) activa los flujos y da coherencia al suministro de materiales e insumos, al manejo de inventarios y a la integración de las cadenas logísticas de producción globalizadas.

Tales puertos concentradores atraen diversas actividades económicas relacionadas no sólo con la transportación y el almacenamiento de la mercancía, sino con el desarrollo de fases específicas del proceso productivo, con la elaboración de estrategias logísticas de valor agregado en las fases de circulación y distribución, con la administración y gestión de inventarios, con la formación de circuitos de comunicación e información, entre otros. Al concentrar actividades y expandir sus zonas de influencia territorial actúan como nodos de amplio alcance territorial, excluyendo, en muchos casos, a puertos tradicionales, asociados normalmente con regiones menos dinámicas o con dificultades para integrarse dentro de la economía-mundo capitalista.

En un proceso de globalización selectiva y excluyente, el desarrollo de puertos concentradores en México es limitado. Dada la densidad de los mercados y el nivel de participación en los flujos de la producción manufacturera mundial, difícilmente podrán desarrollarse concentradores globales en los litorales nacionales. En el mejor de los casos podrán formarse hubs regionales o intermedios en aquellas zonas donde se crucen las embarcaciones que navegan en los ejes este-oeste del hemisferio norte con las que se desplazan por las rutas norte-sur. 
Es precisamente en el Pacífico mexicano donde este proceso empieza a tomar forma. Actualmente, tanto Manzanillo como Lázaro Cárdenas muestran características propias de puertos concentradores intermedios dentro de la red global de transporte multimodal, aprovechando las dos vertientes para concentrar un mayor número de toneladas de carga movida por contenedores en el litoral.

Por un lado, la expansión de sus redes multimodales de trenes de doble estiba les ha permitido alcanzar a más territorios del interior del país. Desde luego, tal proceso de expansión y consolidación del hinterland nacional pasa por la mejora en la eficiencia de sus conexiones intermodales desde el litoral hacia ciudades medias y grandes del occidente, el centro, el Bajío y el norte del país, basadas tanto en la reducción de las demoras y elevados tiempos de estadía de los flujos en los nodos portuarios, como en el desarrollo de servicios de doble estiba de contenedores con mayor confiabilidad en los tiempos de entrega de la mercancía que permitan un flujo continuo y ágil de las cadenas de suministro entre los puertos y las zonas del interior.

Por otro, desde 2005 ambos puertos comenzaron a realizar actividades de transbordo de contenedores procedentes del Lejano Oriente, cuyo destino no era el territorio nacional, sino los países del litoral del Pacífico de Centro y Sudamérica. Por ello, su jerarquía dentro de la red global ha crecido en la medida que se constituyen también como nodos claves para articular las cadenas de suministro continentales, además de atender los requerimientos nacionales.

Finalmente, del análisis previo se desprende que la mayoría de los puertos del Pacífico tendrán funciones alimentadoras dentro de la red global de transporte intermodal. Ahora bien, hay alimentadores, como Ensenada, Mazatlán, Salina Cruz y Puerto Chiapas que están encontrando su nicho de mercado y operadores eficaces para estructurar las conexiones intermodales requeridas. En este sentido, tienen una posibilidad para consolidar su posición de alimentadores eficaces e incluso escalar, en el mediano plazo, hacia niveles de mayor relevancia en el ámbito nacional. Sin embargo, hay alimentadores en situación de estancamiento durante las últimas décadas, que podrían quedar excluidos de la red, a menos que se desarrollen políticas públicas específicas para revertir la situación actual (como el caso de los puertos del Istmo de Tehuantepec) para que encuentren nichos específicos en los que puedan ser competitivos y logren concitar el concurso de actores relevantes en la formación de redes multimodales nacionales e internacionales, a través de esquemas de planeación para alcanzar un desarrollo regional incluyente, 
que impulse, entre otros aspectos, una eficaz integración modal, logística y territorial de los litorales.

\section{Referencias}

Autoridad Marítima de Panamá (AMP). (2019) Cuadros Boletín Estadístico Marítimo Portuario. Recuperado de https://amp.gob.pa/wp-content/ uploads/2019/07/CUADROS-BOLETIN-ESTADISTICO-ENERO-DICIEMBRE-2017-2018.pdf

Banco Mundial (BM). (2019). PIB (US\$ a precios actuales). Recuperado de http://datos.bancomundial.org/indicador/NY.GDP.MKTP.CD

Castells, M. (2006). La Sociedad Red. 2da edición, Madrid: Alianza Editorial

Castells, M. (2010). Globalisation, Networking, Urbanisation: Reflections on the Spatial Dynamics of the Information Age. Urban Studies, 47(13), 2737-2745. doi: https://doi.org/10.1177/0042098010377365

Cedillo, M., Lizarraga, G., \& Martner, C. (2019). Medición de la fluidez en corredores de transporte de carga intermodal. (Publicación Técnica 544) Instituto Mexicano del Transporte. Recuperado de https:/imt.mx/archivos/ Publicaciones/PublicacionTecnica/pt544.pdf

Conferencia de las Naciones Unidas sobre Comercio y Desarrollo (UNCTAD) (1992). La comercialización del puerto y las perspectivas del puerto de tercera generación. Comisión del Transporte Marítimo. Ginebra: Naciones Unidas.

Containerisation International (2014). The Box Benchmark. London: Informa Business.

Coordinación General de Puertos y Marina Mercante (CGPMM). (2020). Informe Estadístico Mensual: Movimiento de Carga, Buques y Pasajeros en los Puertos de Mexico. (Enero - diciembre, 2018-2019). Recuperado de http://www.sct.gob.mx/fileadmin/CGPMM/U_DGP/estadisticas/2019/ Mensuales/12_diciembre_2019.pdf

Fossey, J. (1997). Relay Gold. Containerisation International, 30 (6), 10-14. Hiernaux, D. (1995). La región insoslayable. Revista EURE-Revista de Estudios Urbano Regionales, 21(63), 33-40. Recuperado de http://www.eure.cl/ index.php/eure/article/view/1136

Hoffmann, J. (2000). El potencial de los puertos pivotes en la costa del Pacífico sudamericano. Revista de la CEPAL, (71), 121-143. Recuperado de https:// repositorio.cepal.org/bitstream/handle/11362/12214/1/071121143_ es.pdf 
Instituto Mexicano del Transporte (IMT-SCT). (2002). Estudio para el desarrollo integral y del transporte en la región del Istmo de Tehuantepec: Corredor Intermodal. México, Querétaro: Instituto Mexicano del Transporte.

Martner, C. (2008). Transporte Multimodal y Globalización en México. México, D.F: Trillas.

Martner, C. (2010). Puertos, espacio y globalización: el desarrollo de Hubs en México. Convergencia, 17(52), 319-360. Recuperado de http://www.scielo.org.mx/scielo.php?script=sci_arttext\&pid=S140514352010000100013\&lng=es\&nrm=iso

Martner, C. (2012). El sur también existe: el corredor multimodal del istmo de Tehuantepec en la era de la globalización. Región y Sociedad, 24(54), 97-134. doi: https://doi.org/10.22198/rys.2012.54.a150

Noteboom, T. (2008). The relationship between seaports and the intermodal hinterland in light of global supply chains: European challenges. (Discussion Paper No. 2008-10). Recuperado de https://www.itf-oecd.org/sites/ default/files/docs/rtapr08notteboom.pdf

O'Kelly, M. (1998). A geographer's analysis of hub-and-spoke networks. Journal of Transport Geography, 6(3), 171-186. doi: https://doi.org/10.1016/j. retrec.2009.12.004

Ohmae, K. (1991). El poder de la triada: las nuevas reglas de la competencia mundial. Madrid: MacGraw-Hill/Interamericana.

Rodrigue, J. P. (2017). The Geography of Transport Systems. New York: Routledge.

Rodrigue, J. P., \& Notteboom, T. (2010). Foreland-based regionalization: Integrating intermediate hubs with port hinterlands. Research in Transportation Economics 27(1), 19-29. doi: https://doi.org/10.1016/j. retrec.2009.12.004

Rodrigue, J. P., \& Notteboom, T. (2011). Port regionalization: improving port competitiveness by reaching beyond the port perimeter. Port technology international, 52, 11-17. Recuperado de https://pdfs.semanticscholar.or g/4f64/20f06815cb246616c39a0f7fea651887cc08.pdf

Rodríguez, H. (2019). El conflicto ambiental en el Istmo de Tehuantepec: Una región en perspectiva histórica. En M. Robledo, H. Reyes, \& O. Reyes (Eds.), La historia ambiental de México: Estudios de caso. (pp. 35-42). San Luis Potosí: Universidad Autónoma de San Luis Potosí. Recuperado de http://sociales.uaslp.mx/Documents/Publicaciones/Libros/LaHistAmbMexico.pdf 
San Martín, J. (1997). Análisis comparativo de competitividad entre los corredores de transporte internacional y el puente transítmico mexicano. Recuperado de https://es.slideshare.net/AcademiaDeIngenieriaMx/anlisis-comparativode-competitividad-entre-los-corredores-de-transporte-internacionalesy-el-puente-transstmico-mexicano

Santos, M. (2000). La Naturaleza del Espacio. Barcelona: Ariel Geografía.

Sassen, S. (2003). Localizando ciudades en circuitos globales. Revista EURE, 29(88), 5-27. doi: http://dx.doi.org/10.4067/S0250-71612003008800001

Segura, J., \& Sorroza, C. (1994). Una Modernización Frustrada (1940-1986). En R. Aoyama (Ed.), Economía Contra Sociedad: el Istmo de Tehuantepec 1907-1986. (pp. 72-84). México, D.F: Nueva Imagen.

Veltz, P. (2006). Entreprises et territoires, entre fragmentation et intégration. En E. Heurgon (Ed.), Le développement durable. Paris: Editions de l'Aube. Veltz, P. (2014). Mondialisation, villes et territoires: Une économie d'archipel, 2e éd. Paris: Quadrige.

Wallerstein, I. (1996). Después del Liberalismo. México, DF: Siglo XXI Editores Wallerstein, I. (2016). El mundo está desencajado. Interpretaciones históricomundiales de las continuas polarizaciones, 1500-2000. Ciudad de México: Siglo XXI editores.

Zanela, A. (2015). Acortarán estadía de contenedores en puertos mexicanos. Recuperado de http://t21.com.mx/maritimo/2015/08/14/acortaranestadia-contenedores-puertos-mexicanos

Zohil, J., \& Prijon, M. (1999). The MED rule: the interdependence of container throughput and transhipment volumes in the Mediterranean ports. Maritime Policy \& Management, 26(2), 175-193. doi: https://doi. org/10.1080/030888399286998 